\title{
EVALUACIÓN DE LA CAPACIDAD FÉRTIL DE LA RETAMA (Spartium junceum)
}

\section{RETAMA CAPACITY ASSESSMENT FERTILE (Spartium junceum)}

\author{
Nicolás A. Román Cabello, Carlos Suasnabar Astete ${ }^{2}$ \\ Instituto de Investigación de la Facultad de Agronomía
}

\section{RESUMEN}

En el presente estudio se han establecido comparaciones entre el modelo estándar de la gametogénesis masculina o microsporogénesis para plantas angiospermas, que sigue una pauta o regla de formación que puede ser monitoreada, y la microsporogénesis en Spartium junceum (retama) Se han seleccionado plantas en diferentes estadios de formación: prefloración, floración y postfloración y anteras en antesis para evaluar tanto la maduración del polen como su viabilidad. Se ha utilizado la metodología de estudios correlacionales y de regresión para evaluar la causalidad y la relación entre las diferentes variables en estudio, para tal efecto se han evaluado las correlaciones entre los diferentes estadios de células que siguen una pauta de proporcionalidad preestablecida y regresiones para predecir el número de células que se forman en cada estadio, además para poder predecir valores esperados entre los diferentes estadios. Asimismo se han utilizado pruebas de ji cuadrado para determinar si las desviaciones entre los valores esperados y los observados corresponden o no a una proporción establecida por el modelo de gametogénesis para las plantas angiospermas. Se ha determinado que Spartium junceum no sigue las pautas del modelo y que las relaciones y proporciones entre los diferentes estadios está alterada y hay una baja producción de polen viable tiene una alta capacidad fértil, lo cual hace suponer que hay una fuerte presión de selección a favor de los granos de polen mejor conformados. El presente estudio corrobora las hipótesis planteadas en los anteriores trabajos con tres especies diferentes (Antirrhinum m., Brassica c. y Zea $\mathrm{m}$.) según las cuales hay una baja de la capacidad fértil en las plantas angiospermas debido a laos factores climáticos cambiantes ante lo cual la planta responde con una selección de su polen a favor de los mejor conformados y viables.

Palabras clave: microporogesis, viabilidad, retama, gametogénesis, polen

\begin{abstract}
In the present study were established comparisons between the standard model of the male gamete or microsporogenesis angiosperm plant, following a training guidelines or rules that can be monitored, and microsporogenesis in Spartium junceum (broom) plants were selected in different training stages: preflowering, flowering and post flowering and anthesis anthers to evaluate both the maturation of pollen viability. It was used the methodology correlational and regression studies to assess the causality and the relationship between different variables under study, for this purpose it was evaluated the correlations between the different stages of cells follow a preset pattern of proportionality and regressions to predict the number of cells in each stage are also expected to predict values between the different stages. Also been used chi-square tests to determine if the deviations between predicted and observed values correspond or not to a target ratio for gametogenesis model for angiosperm plants. It has been determined that Spartium junceum not follow the guidelines of the model and relations and proportions between the different stages is impaired and there is a low production of viable pollen has a high fertile, which suggests that there is strong selection pressure for better pollen grains formed. This study supports the hypothesis proposed in the previous works with three different species (Antirrhinum m., Brassica c., Zea m.) According to which there is a low reproductive capacity in angiosperm plants due to changing climatic laos at what which the plant responds with a selection of their pollen in favor of better shaped and viable.
\end{abstract}

2 manuelalomia38@hotmail.com 
Key words: microporogesis, viability, broom, gametogenesis, pollen

\section{INTRODUCCIÓN}

Durante el ciclo biológico de las plantas angiospermas, se produce la gametogénesis que es un evento a partir del cual no solamente se forman los gametos, tanto masculinos como femeninos, sino que se produce la recombinación de genes durante la Profase primera de la meiosis mediante el denominado "crossing over" o entrecruzamiento de cromosomas homólogos. La microsporogénesis, que es la formación del polen, es decir de los gametos masculinos sigue un proceso durante el cual suceden dos divisiones sucesivas, una de ellas reduccional y otra ecuacional, el resultado del cual es la formación de cuatro gametos viables con tres núcleos incluidos en cada uno de ellos. Lo que hace interesante este proceso es que estas divisiones sucesivas ocurren con una regla de formación predecible, de tal modo que estudiando la cantidad de células que se producen en cada una de estas divisiones (valores observados) se puede determinar la cantidad de polen que pueda potencialmente producirse (valores esperados). La desviación de estos valores observados con los esperados nos dará una pauta para determinar la capacidad fértil de la planta y en consecuencia también el grado de androesterilidad, lo cual se puede someter a un análisis matemático y estadístico de tal modo que obteniendo un modelo matemático de la microsporogénesis podríamos eventualmente predecir el número de granos de polen que se formarán lo cual será utilizado como indicadores de la calidad fértil de la planta. Esto nos puede conducir más adelante a desarrollar hipótesis sobre la capacidad de sobrevivencia, resistencia de la planta ante factores adversos: ataque de plagas y enfermedades, factores climáticos etc. y las interacciones que ocurren en la naturaleza con respecto a esta planta. La retama se estudia en este trabajo ya que su supervivencia en la localidad estuvo en riesgo aunque hasta ahora no se sabe bien qué factores adversos la pusieron en esta situación. Los estudios previos en Antirrhinum majus ("dogo") Brassica campestris ("yuyo"), y Zea mays (maíz) han demostrado que la androesterilidad puede ocurrir ya en algunas de las etapas de la microsporogénesis (microsporócitos) y reducir la capacidad fértil de las plantas. En el caso de Brassica y Antirrhinum no se cumple necesariamente el modelo preestablecido y en maíz se ha observado que esta planta está reduciendo drásticamente su fertilidad masculina con el paso del tiempo. Con estas consideraciones y los antecedentes de estudios previos el presente trabajo tiene como objetivos evaluar la capacidad fértil de los granos de polen de Spartium junceum ("retama") y desarrollar un modelo matemático predictivo para determinar la cantidad de polen potencial que pueda producir esta planta, cuya desviación permita diseñar a su vez modelos de capacidad fértil masculina para otras especies.

\section{MATERIALES Y MÉTODOS}

El presente trabajo se realizó en el laboratorio del Instituto de Biotecnología Ingeniería Genética de la Universidad Nacional del Centro del Perú, Área de Citogenética. De treinta plantas ubicadas en las localidades de Pilcomayo, y Ocopilla se tomaron muestras de las inflorescencias de plantas en diferentes estadios de floración, tomando como referencia anteriores investigaciones, de las cuales se tomaron las siguientes muestras:

M1: Prefloración: 25 Anteras inmaduras en meiosis

M2: Floración: 10 Anteras con polen maduro

M3: Post floración 110 Anteras con polen en sacos polínicos

M4: Post floración 210 Anteras maduras antes de la antesis.

La metodología consistió en evaluar la relación causal (causalidad) entre dos variables (valores observados de número de granos de polen viable, " $X$ " y valores esperados de número de granos de polen viables, "Y") para el modelo de gametogénesis establecido 0 estándar en angiospermas; la diferencia entre estas variables medida estadísticamente dará una aproximación (matemática) de la capacidad fértil, masculina de Spartium junceum estableciéndose así un modelo para medir la capacidad fértil del polen en la planta en estudio. Se utilizó la técnica de 
conteo de estructuras gaméticas previamente teñidas y sometidas a "squash" observadas al microscopio y contabilizadas según el campo visual promedio. Para el conteo se utilizó el método de Breed, cámara de Neubauer, y la fotomicrografía. Para la colección del material se tomó en cuenta la madurez sexual de la planta. La preservación de las anteras se realizó en carnoy 3:1 (3 de alcohol de 960 y uno de ácido acético glacial) en frascos etiquetados y refrigerados a $40 \mathrm{C}$., para la observación y conteo al microscopio se utilizó el método de Breed, una cámara de Neubauer y el campo visual promedio (CVP): Se observó a $3,2 \mathrm{X}, 100 \mathrm{X}$ y $400 \mathrm{X}$ (para observar eventos meióticos que permitan diferenciar la célula madre del polen de los microsporócitos primarios). Para las evaluaciones se utilizaron correlaciones, regresiones lineales y pruebas de ji cuadrado. Los equipos utilizados fueron: Microscopio Laboval 4 con cámara fotográfica incorporada, cámara fotográfica AGFA DC-1033 m de $1^{\circ} \mathrm{mpx}$, frízer, refrigeradora, cámara de Neubauer; los materiales fueron: frascos de vidrio (20), placas de petri, tubos eppendorf, estiletes, placas porta y cubre objetos, vacutainers (10), Pizeta, vaso de precipitación y mechero de alcohol. Los reactivos fueron: Clorox 1:9, alcohol de 960, ácido acético glacial y los colorantes orceína y carmín acéticos, verde brillante, violeta genciana, fucsina ácida y rubín.

\section{RESULTADOS Y DISCUSIÓN}

Cuadro 1. Correlaciones entre las estructuras gaméticas

\begin{tabular}{|c|c|c|c|c|c|c|}
\hline & CMP & $\mathrm{MP}$ & $\mathrm{MS}$ & $\mathrm{M}(\mathrm{T})$ & $\mathrm{P} 1$ & $\mathrm{P} 2$ \\
\hline CMP & & 0,2481 & 0,2611 & 0,3221 & 0,3412 & 0,623 \\
\hline MP & & & 0,3684 & 0,0029 & 0,4312 & 0,6833 \\
\hline MS & & & & 0,4162 & 0,368 & 0,5311 \\
\hline$M(T)$ & & & & & 0,2112 & 0,4802 \\
\hline P1 & & & & & & 06344 \\
\hline P 2 & & & & & & \\
\hline
\end{tabular}

Cuadro 2. Regresiones entre las estructuras genéticas

\begin{tabular}{|c|c|c|c|c|c|c|}
\hline & CMP & MP & MS & $\mathrm{M}(\mathrm{T})$ & P1 & P2 \\
\hline CMP & & 0,7661 & 0,6882 & 0,2213 & 0,083 & 0,9868 \\
\hline MP & & & 0,0124 & 0,0044 & 4,326 & 2,2911 \\
\hline MS & & & & 0,4621 & 0,042 & 0,0033 \\
\hline$M(T)$ & & & & & 1,014 & 0,331 \\
\hline $\mathrm{P} 1$ & & & & & & 0,3612 \\
\hline P 2 & & & & & & \\
\hline
\end{tabular}

Cuadro 3. Ji cuadrado entre las estructuras genéticas.

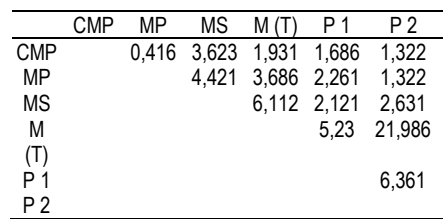

\section{DESCRIPCIÓN DE SÍMBOLOS}

CMP: Células madre del polen

MP: Microsporocitos primario

MS: Microsporocitos secundario

$M(T)$ : Tétradas o microsporas

P1: Polen inmaduro (primario)

P2: Polen maduro

Cuadro 4. Amplitud del stok de polen viable.

\begin{tabular}{lllll}
\hline & $\begin{array}{c}\text { Polen } \\
\text { normal }\end{array}$ & $\begin{array}{l}\text { Polen } \\
\text { estéril }\end{array}$ & Total & \multicolumn{1}{c}{$\%$} \\
\hline CVP $_{1}$ & 82 & 4 & 86 & 95,34 \\
$\mathrm{CVP}_{2}$ & 91 & 6 & 97 & 93,81 \\
$\mathrm{CVP}_{3}$ & 6 & 6 & 70 & 91,42 \\
$\mathrm{CVP}_{4}$ & 106 & 3 & 109 & 92,247 \\
$\mathrm{CVP}_{5}$ & 120 & 2 & 122 & 98,36 \\
$\mathrm{CVP}_{6}$ & 88 & 4 & 92 & 95,65 \\
$\mathrm{CVP}_{7}$ & 96 & 3 & 90 & 96,96 \\
$\mathrm{CVP}_{8}$ & 94 & 3 & 94 & 96,90 \\
$\mathrm{CVP}_{9}$ & 130 & 2 & 132 & 98,48 \\
$\mathrm{CVP}_{10}$ & 84 & 3 & 87 & 96,551 \\
\hline
\end{tabular}

$X=95,691 \%$

CVP $=$ Campo visual promedio

Cuadro 5. Escala propuesta para evaluar la amplitud del stok de polen viable

\begin{tabular}{ll}
$0-20 \%$ & Amplitud restringido \\
$21-40 \%$ & Amplitud baja \\
$41-50 \%$ & Amplitud media \\
$51-70 \%$ & Amplitud buena \\
$71-80 \%$ & Amplitud muy buena \\
Mayor de $80 \%$ & Fertilidad total \\
\hline
\end{tabular}

El proceso de microsporogénesis en Sparteum junceum. como en cualquier otra planta angiosperma puede ser monitoreado debido a que presenta una secuencia meiótica bastante predecible ya que sigue un orden de formación 
(esto es muy cierto para todas las especies donde existe recombinación genética, incluyendo las especies animales): esta secuencia predice relaciones de 1:1 como entre las células madres del polen y los microsporócitos primarios, de 1:2 entre microsporócitos primarios y microsporócitos secundarios 0 de 1:4 como entre célula madre del polen y microsporas y granos de polen etc. Cuando estas proporciones esperadas se desvían de las proporciones observadas (nos estamos refiriendo a desviaciones de tipo estadístico que pueden ser determinadas con cierta certeza y margen de error) entonces se puede decir que la especie tiene "problemas" en la formación de sus gametos masculinos o sea androesterilidad, que pueden ser atribuidas a diferentes causas que luego pueden ser estudiadas

El análisis estadístico de correlación evidencia la relación proporcional (o no) entre estas estructuras gaméticas, y el de regresión cuantifica el aumento o disminución del número de células de un determinado tipo, cuando aumenta o disminuye en una unidad (1) el número de células de otro tipo, por ejemplo microsporócitos secundarios y microsporas. La prueba de chi cuadrado o X2 ha sido utilizada para determinar si las desviaciones entre las proporciones observadas y las esperadas corresponden 0 no a una determinada hipótesis: $1: 1,1: 2,1: 4$ etc. y esto es útil ya que permite determinar con mucha precisión la androesterilidad.

La amplitud del stock de polen viable ha permitido determinar que el polen que se forma, independientemente de si hay o no problemas en la microsporogénesis, es viable, o a la inversa si no lo es y en qué cantidad, es decir la viabilidad se cuantifica para conectar esta situación con las pruebas estadísticas arriba mencionadas.

En esta discusión hay que decir que las investigaciones que se hicieron (incluyendo tres trabajos anteriores en otras especies) tuvieron la pretensión de seguir hechos de la naturaleza utilizando modelos matemáticos, es decir formalizar hechos reales que no necesariamente siguen una pauta predeterminada ni predecible por lo que es necesario indicar que las pautas estadísticas obtenidas tienen un grado de confiabilidad, pero también un grado de no confiabilidad ya que en el estudio de estos fenómenos biológicos implica la participación de muchas variables e incluso de variables ocultas, que indican nuestra ignorancia con respecto a lo que realmente sucede. De este modo los resultados son parciales hasta que no se conozcan más variables

$\mathrm{Al}$ evaluar las correlaciones entre las diferentes estructuras gaméticas se puede observar que no existen correlaciones entre ellas. Esto indica una formación irregular de las estructuras gaméticas que no sigue las pautas preestablecidas para este tipo de modelos de microsporogénesis de las angiospermas: Así la relación entre CMP y MP ( $r=02481)$ CMP y MS ( $r=0.261)$ CMP y $T(r=0,3412)$ CMP y P1 $(r=0.3412)$ en donde las correlaciones son muy bajas pueden estar indicando una serie de eventos meióticos muy irregulares y esto puede tener muchas causas, desde bajo nivel de fitohormonas hasta factores de índole climático y ambiental (desde la perspectiva genética). Incluso donde se observa la "mejor" relación para el caso de CMP y P2 que es r=0,623solamente el 37,44 de la relación es atribuible a la meiosis normal, mientras que hay una indeterminación de $72.52 \%$ que indica que hay "variables ocultas" que distorsionan la relación y la proporción gamética, $Y$ esto se repite para las demás correlaciones entre las estructuras gaméticas.

Está claro que de estas formalizaciones no se puede deducir aún que existan problemas de fertilidad en las plantas de retama ya que es necesario referirse al stock de polen viable que es de $95.68 \%$. ¿Cómo comprender estos resultados? Parece que este stock tan alto representa solamente al polen ya formado, que aunque sea de manera irregular y en baja cantidad, sin embargo presenta una alta tasa de viabilidad, y se debe a un proceso de selección gamética es decir sobreviven los granos de polen más aptos.

En el cuadro de regresiones se observa que al igual que en las investigaciones anteriores (con Antirrhinum m. Brassica c. y Zea m.) existen valores que pueden ser utilizados como indicadores de la producción de microsporócitos, microsporas y polen a partir de las células madres del polen.

Así por cada 100 CMP que se produzcan habrá alrededor de 89 granos de polen $(b=08.868)$ que como ya vimos son en su mayoría viables y la diferencia (pues deberían haber unos 400 granos de polen) se explica por alguna falla en la meiosis 
justo cuando los MS deberían pasar a microsporas (Il división meiotica, profase y/o metafase) y luego a polen (telofase II) y esto se evidencia por la gran distorsión de los valores de regresión entre CMP y Microsporas $(b=0.2213)$. De aquí se deduce que las regresiones nos pueden indicar en qué momento se realiza u ocurren desfases en el proceso meiótico. Si se sigue estudiando el cuadro $\mathrm{N}^{\circ} 2$ de valores de regresión veremos que como en el caso anterior existen "distorsiones" meióticos entre MP y Microsporas (profase I y profase II), entre MS y Polen inmaduro (P1) y maduro (P2) (profase II y telofase II) etc. Y Las causas de estas distorsiones deben explicarse a nivel de proceso meiotico, con los resultados de la prueba de ji cuadrado, como se verá acontinuación tiene sus propias y particulares pautas.

Para el caso de CMP y MP el valor de ji cuadrado $(X 2=0.416)$ nos está indicando que se ha dado la relación 1:1 esperada para este caso y las diferencias no son significativas (se deben al azar). Sin embargo al ver los valores de X2 para todos los otros casos las proporciones están invariablemente sesgadas y/o no corresponden a los valores esperados para estos casos de microsporogénesis en angiospermas y esto corrobora y está en consenso con los otros análisis realizados.

En general se puede decir que en vista de que el proceso de microsporogénesis observado en retama no sigue las pautas esperadas para este modelo de angiospermas, entonces hay una fuerte presunción de esterilidad masculina, y cuando se analizan los otros casos de plantas investigados se puede establecer (hasta ahora) que hay una baja de fertilidad masculina observada al menos en estos hasta ahora cuatro casos investigados con la misma metodología, y como ya se ha explicado anteriormente es que las especies en general están sometidas a variaciones climáticas no previsibles, cambiantes con fuertes bajas de temperatura y sequías agobiantes que ya no siguen un calendario determinado, son más sometidas también al ataque de plagas, enfermedades e incluso virus; todo esto se puede resumir diciendo que hay una sobreexposición a estrés ambiental.

Las evidencias hasta ahora mostradas nos permiten decir que pareciera que existe una baja en la producción de polen en las plantas (por lo menos en las estudiadas) causadas por variaciones extremas ambientales y lo que está favoreciendo un proceso de adaptación en las plantas (el polen que se produce es altamente viable (muy buena amplitud en el stock de polen viable) adaptación que se observa por la presión de selección a favor de los granos de polen bien conformados, que aunque en poca cantidad son muy viables y estamos ante el caso que una meiosis irregular se compensa con una mejor calidad de polen, una situación que en el anterior trabajo con maíz era una suposición que ahora se ve reforzada por estos nuevas evidencias

\section{CONCLUSIONES}

- Sparteum junceum no sigue las pautas y proporciones establecidas para los modelos de microsporogénesis en angiospermas.

- La producción de polen maduro es muy baja y no guarda relación con el número de células madres del polen que se producen y entre estos dos estadios también hay variaciones que no corresponden a las proporciones esperadas para este tipo de procesos.

- El polen inmaduro (P1) pasa normalmente al siguiente estadio ( $\mathrm{P} 2$ ) sin ninguna variación significativa en la proporción 1:1 esperada para este tipo de proceso significativamente de la proporción esperada.

- Las variaciones más significativas entre estadios de la microsporogénesis se pueden relacionar al estudiar las regresiones con variaciones que en realidad existen a nivel de los procesos meióticos (profase I y profase II, profase II y telofase II etc.).

- El polen que se produce, sí bien no en la cantidad adecuada pues hay una reducción bien drástica, sin embargo es por entero viable y esto se corrobora al evaluar la amplitud del stock de polen viable que supera fácilmente el $95 \%$ de viabilidad.

- Se puede deducir de que si bien la microsporogénesis es irregular y no sigue el modelo preestablecido para las plantas angiospermas, el polen producido es viable en su casi totalidad esto es un indicativo de una fuerte presión selectiva a favor del polen mejor conformado y viable lo cual a su vez nos 
indica una eficaz respuesta a las condiciones ambientales y climáticas muy cambiantes que predominan en la zona.

- Con evidencias de anteriores trabajos en otras especies (Antirrhinum m. Brassica c y Zea m.) se puede decir que existe una drástica disminución en la capacidad de producción del polen que estaría ocurriendo por las condiciones cambiantes del clima, ante lo cual las especies (por lo menos las estudiadas hasta ahora) responden con una fuerte presión selectiva a favor de los granos de polen mejor conformados y viables

\section{REFERENCIAS BIBLIOGRÁFICAS}

1. Tamarin E. J. Genética. Ed. CECSA. Madrid España, 1999.

2. Galindo M. Microsporogénesis de alcachofa Cynara scolymus, 2004.

3. ROMAN N. Evaluación a nivel celular de la capacidad fértil de maíz Zea mays. Informe final. Instituto de investigación FAG-UNCP, 2010.

4. CURTIS P. J. Microtecnia vegetal. Ed. Trillas, México D. F., 2008. 